\title{
ON FIBERING SPHERES BY TORUSES
}

\author{
B. ECKMANN, H. SAMELSON AND G. W. WHITEHEAD
}

1. Introduction. In this note we answer a question which was recently asked by $\mathrm{H}$. Hopf, namely whether there exist fiber decompositions of the $n$-dimensional sphere $S^{n}$, or of the $n$-dimensional Euclidean space $E^{n}$, with an $s$-dimensional torus $T^{s}$ (the product of $s$ factors $S^{1}$ ) as fiber. The answers are given in Theorems 1 and 2 below. The fiber decompositions are understood in the sense of fiber bundle [1], ${ }^{1}$ with the group of all homeomorphisms as structure group, that is, we are given three spaces $X, F$ and $M$ (the bundle, the fiber and the base space), and a map $\phi: X \rightarrow M$, the projection; and for every point $p$ of $M$ there is given a neighborhood $V_{p}$ of $p$ and a homeomorphism of $\phi^{-1}\left(V_{p}\right)$ with the product $V_{p} \times F$, such that $\phi^{-1}(q)$ is mapped onto $q \times F$ for every point $q \in V_{p}$. The sets $\phi^{-1}(p)$ are called the fibers.

2. Results. We now consider the case where $X$ is either $S^{n}$ or $E^{n}(n>0)$, and where $F$ is $T^{s}(s>0)$. We shall establish the following results:

TheOREM 1. A fiber decomposition (in the sense of \$1), with a (locally finite) polyhedron as base space, of the $n$-sphere $S^{n}$ with the s-torus $T^{s}$ as fiber exists if and only if $n$ is odd and $s=1$.

TheOREM 2. For no $n$ and $s$ does there exist a fiber decomposition (in the sense of \$1), with a (locally finite) polyhedron as base space, of Euclidean $n$-space $E^{n}$ with the s-torus $T^{s}$ as fiber.

REMARK. We show in $\S 7$ (a) that Theorem 1 holds also if arbitrary separable metric spaces (instead of polyhedra only) are admitted as base spaces. The corresponding statement for Theorem 2 can be made only modulo a theorem concerning singular homology groups, which is probably true, but for which, as far as the authors are aware, there is no proof in the literature.

We note for the if part of Theorem 1 that there exists a well known fiber decomposition of $S^{2 l+1}$ with circles $T^{1}$ as fiber; the base space is the complex projective space $K^{l}$ (of dimension $2 l$ ) (see $[2, \mathrm{p} .55]$ ).

The proofs of Theorems 1 and 2 are based on the consideration of 1948.

Presented to the Society, September 4, 1948; received by the editors March $18^{\circ}$

1 Numbers in brackets refer to the list of references at the end of the paper. 
homotopy groups and singular homology groups and their connections, in particular on the results of Hurewicz-Steenrod [3], Eckmann [4] and Eilenberg-MacLane [5].

3. Homotopy groups. Let $(X, F, M, \phi)$ be a fiber bundle as in $\$ 1$. With a fixed fiber $F_{0}$ and base point $q_{0}$ in $F_{0}$ we form the absolute homotopy groups $\pi_{k}\left(X, q_{0}\right)$ or in short $\pi_{k}(X)$, and the relative homotopy groups $\pi_{k}\left(X, F_{0}, q_{0}\right)$ or in short $\pi_{k}\left(X, F_{0}\right)$; we set up the homotopy sequence of $X \bmod F_{0}$ [6, p. 463]:

$$
\begin{aligned}
\cdots \pi_{k}\left(F_{0}, q_{0}\right) & \rightarrow \pi_{k}\left(X, q_{0}\right) \rightarrow \pi_{k}\left(X, F_{0}, q_{0}\right) \rightarrow \pi_{k-1}\left(F_{0}, q_{0}\right) \\
& \rightarrow \cdots \rightarrow \pi_{1}\left(X, q_{0}\right) .
\end{aligned}
$$

This is an exact sequence; the kernel of each homomorphism is the image under the preceding homomorphism. Furthermore, from Steenrod's results in $[1$, Theorem 9, p. 302] on the existence of a covering homotopy in fiber bundles, and from the reasoning of [3, Theorem 2], or from $\left[4\right.$, p. 162, (4)], it follows that the groups of $X \bmod F_{0}$ and the absolute groups of $M$ are isomorphic (we put $\left.p_{0}=\phi\left(q_{0}\right)\right)$ :

$$
\pi_{k}\left(M, p_{0}\right) \approx \pi_{k}\left(X, F_{0}, q_{0}\right) .
$$

We now consider the special case of Theorem 1 and assume $F=T^{*}$ and $X=S^{n}$ (for the time being we make the restriction $n>2$ ); we denote the base space by $M_{1}$ (by hypothesis, $M_{1}$ is a polyhedron). The first step in the proof of Theorem 1 is the determination, in equations (5), of the homotopy groups of $M_{1}$ up to dimension $n-1$.

Let $Z_{j}$ stand for the free abelian group of $j$ generators. The following facts are well known:

$$
\begin{array}{lr}
\pi_{1}\left(T^{s}\right)=Z_{s}, & \\
\pi_{k}\left(T^{s}\right)=0 & \text { for } k \neq 1 ; \\
\pi_{k}\left(S^{n}\right)=0 & \text { for } 0<k<n .
\end{array}
$$

The connectedness of $T^{s}$ implies that $\pi_{1}\left(M_{1}\right)$ vanishes: every closed path in $M_{1}$ is, by the covering homotopy theorem, image of a path in $S^{n}$ with initial and terminal point in the same fiber, and therefore also image of a closed path in $S^{n}$; moreover $\pi_{1}\left(S^{n}\right)$ vanishes. For $2 \leqq k<n$ it follows from the exactness of (1) and from (4) that the groups $\pi_{k}\left(S^{n}, T_{0}^{s}\right)$ and $\pi_{k-1}\left(T^{s}\right)$ are isomorphic (if in a "section of length 4" in an exact sequence the two outer groups vanish, then the two inner groups are isomorphic, cf $[7, \mathrm{p} .687])$. By (2), the groups $\pi_{k}\left(S^{n}, T_{0}^{s}\right)$ and $\pi_{k}\left(M_{1}\right)$ are isomorphic. It follows that $\pi_{k}\left(M_{1}\right)$ and $\pi_{k-1}\left(T^{s}\right)$ are isomorphic for $2 \leqq k<n$; in view of (3) we can therefore 
state the following equations:

$$
\begin{aligned}
& \pi_{k}\left(M_{1}\right)=0 \quad \text { for } 0<k<n, k \neq 2, \\
& \pi_{2}\left(M_{1}\right)=Z_{s} .
\end{aligned}
$$

4. Homology groups. Let $H^{i}$ be the $i$ th singular integral homology group, and let $\Sigma^{i}$ be the subgroup of spherical cycles. To prove our Theorems 1 and 2 we shall make use of Theorem II $^{m}$ of EilenbergMacLane [5] which says: If $X$ is a space such that $\pi_{i}(X)=0$ for $i<m$ and $m<i<n(m<n)$ then $H^{i}(X)$, for $i<n$, and $H^{n}(X) / \Sigma^{n}(X)$ are determined by the structure of the group $\pi_{m}(X)$; our equations (5) show that this theorem is applicable to the space $M_{1}$ with $m=2$. In order to compute the groups $H^{i}\left(M_{1}\right), i<n$, and $H^{n}\left(M_{1}\right) / \Sigma^{n}\left(M_{1}\right)$, we use a geometrical method; we construct a space $P$ whose homotopy groups $\pi_{i}(P)$ for $i<n$ are isomorphic with the corresponding groups of $M_{1}$, and whose homology groups are known; by the theorem just mentioned the groups of $M_{1}$ will be isomorphic to those of $P$, for the appropriate dimensions. The space $P$ is defined as the topological product of $s$ complex projective spaces $K^{l}$, with $l$ sufficiently large, for example, $l>n$. For the homotopy groups of $K^{l}$ the following relations are known:

$$
\pi_{2}\left(K^{l}\right)=Z_{1} ; \quad \pi_{i}\left(K^{l}\right)=0, \quad 0<i<2 l+1, i \neq 2 .
$$

These relations are immediate consequences of equation (5), for $n=2 l+1$ and $s=1$, since, as mentioned in $\$ 2, K^{l}$ is the base space of a fiber decomposition of $S^{2 l+1}$ with fiber $T^{1}$. The homotopy groups of a topological product are the direct sums of the homotopy groups of the factors; the relations

$$
\pi_{i}(P) \approx \pi_{i}\left(M_{1}\right), \quad 0<i<n
$$

follow then from (5) and (6).

The homology groups of $K^{l}$ are given by the equations [8, p. 83]: (8) $H^{2 k}\left(K^{l}\right)=Z_{1}$, for $0 \leqq k \leqq l ; H^{2 k-1}\left(K^{l}\right)=H^{2 l+k}\left(K^{l}\right)=0$ for $k>0$. The subgroups of spherical cycles are given by

$$
\Sigma^{2}\left(K^{l}\right)=H^{2}\left(K^{l}\right) ; \quad \Sigma^{i}\left(K^{l}\right)=0, \quad i \neq 2,
$$

as can be seen easily from (6) and from the fact that the generators of $H^{2}\left(K^{l}\right)$ and of $\pi_{2}\left(K^{l}\right)$ are both given by the subspace $K^{1}$ of $K^{l}$. The homology groups of $P$ can now be found from (8) by Künneth's theorem; we note that all groups of odd dimensions vanish, that the groups of even dimensions (not greater than $\operatorname{dim} P$ ) do not vanish, and that nonbounding spherical cycles exist only in dimension 2 : 


$$
\begin{aligned}
& H^{2 t-1}(P)=0, t>0 ; H^{2 t}(P) \\
& \Sigma^{t}(P) \neq 0 \text { for } 0 \leqq t \leqq 2^{-1} \operatorname{dim} P ; \\
& \text { for } t \neq 2 .
\end{aligned}
$$

5. Proof of Theorem 1. As noted above, equations (5) and (7) make it possible to apply Theorem $\mathrm{II}^{m}$ of [5] with $m=2$; the following relations between the homology groups of $M_{1}$ and $P$ hold therefore:

$H^{i}\left(M_{1}\right) \approx H^{i}(P)$ for $0 \leqq i<n ; H^{n}\left(M_{1}\right) / \Sigma^{n}\left(M_{1}\right) \approx H^{n}(P) / \Sigma^{n}(P)$.

This amounts to a partial determination of the homology groups of $M_{1}$. On the other hand, the homology groups of $M_{1}$ vanish for dimensions above $n-s$ : since every point $p$ of $M_{1}$ has a neighborhood $V$ such that $V \times T^{s}$ is homeomorphic with the open subset $\phi^{-1}(V)$ of $E^{n}$ it is clear that the dimension of the polyhedron $M_{1}$ is $n-s$, and therefore the homology groups in higher dimensions vanish. We state the equation:

$$
H^{i}\left(M_{1}\right)=0 \quad \text { for } i>n-s .
$$

Theorem 1 follows now quickly from the properties of the homology groups of $M_{1}$, given by (10), (11), (12):

By equation (12), the group $H^{n}\left(M_{1}\right)$ vanishes. From (11) we see that therefore the subgroup $\Sigma^{n}(P)$ equals the whole group $H^{n}(P)$. Comparing this with (10) we conclude that $n$ must be odd, since obviously $n<\operatorname{dim} P$ (notice that we assumed $n>2$ ). But then $H^{n-1}(P)$ does not vanish, again by (10). By (11) $H^{n-1}\left(M_{1}\right)$ does not vanish; by (12) we have $n-1 \leqq n-s$; it follows that $s=1$. This concludes the proof of Theorem 1 for $n>2$. For $n=2$ the only case of interest is $s=1$; it is easily seen that then the Jordan curve theorem for $S^{2}$ would imply that every point of the compactum $M_{1}$ is a cut point, which is impossible [9, p. 54]. For $n=1$ there is nothing to prove.

6. Proof of Theorem 2. We now assume that we have a fiber decomposition of $E^{n}(n>0)$ with fiber $T^{s}(s>0)$; we denote the base space by $M_{2}$; by hypothesis, $M_{2}$ is a polyhedron. The reasoning which leads to a contradiction is similar to that of $\S 5$. Equation (4) is replaced by

$$
\pi_{k}\left(E^{n}\right)=0,
$$$$
k>0 \text {. }
$$

$\pi_{1}\left(M_{2}\right)$ vanishes, for the same reason as $\pi_{1}\left(M_{1}\right)$. The exactness of $(1)$, and $\left(4^{\prime}\right)$ show now that $\pi_{k}\left(E^{n}, T_{0}^{s}\right)$ and $\pi_{k-1}\left(T_{0}^{s}\right)$ are isomorphic for all $k \geqq 2$. We find, therefore, applying (2) and (3),

$$
\pi_{2}\left(M_{2}\right)=Z_{s}, \quad \pi_{k}\left(M_{2}\right)=0 \quad \text { for } k \neq 2 .
$$

We again consider the comparison space $P$, the product of $s$ factors 
$K^{l}$, with $l>n$. From $\left(5^{\prime}\right)$ and (6) it follows, as in $\$ 4$, that

$$
\pi_{i}(P) \approx \pi_{i}\left(M_{2}\right) \quad \text { for } i<2 l+1 ;
$$

Theorem II ${ }^{m}$ of [5], which applies again with $m=2$ because of $\left(5^{\prime}\right)$ and $\left(7^{\prime}\right)$, shows that

$$
H^{i}(P) \approx H^{i}\left(M_{2}\right) \quad \text { for } i<2 l+1 .
$$

By the reasoning which we used to establish (12), we see that the dimension of $M_{2}$ is $n-s$ and that the homology groups in dimensions above $n-s$ vanish:

$$
H^{i}\left(M_{2}\right)=0 \quad \text { for } i>n-s .
$$

We now choose an even $i_{0}$ with $n-s<i_{0}<2 l+1$; this is possible since $l>n$; from (10) we see that $H^{i_{0}}(P)$ does not vanish, but $H^{i_{0}}\left(M_{2}\right)$ vanishes because of $\left(12^{\prime}\right)$. This contradiction establishes Theorem 2 .

7. Remarks. (a) It would be desirable to prove Theorems 1 and 2 with arbitrary separable metric spaces (and not only polyhedra) as admissible base spaces for the fiber decompositions. Now the only point where the polyhedral character of $M_{1}$ and $M_{2}$ has been made use of is in establishing equation (12) and $\left(12^{\prime}\right)$. We shall show how to derive (12), and so to prove Theorem 1 , if $M_{1}$ is assumed to be only separable metric: $M_{1}$ is compact, as image of $S^{n}$. Consider a neighborhood $V_{p}$ of a point $p \in M_{1}$, for which $V_{p} \times T^{s}$ is homeomorphic with $\phi^{-1}\left(V_{p}\right)$, under a homeomorphism $h$. Since $T^{s}$ is a product of one-dimensional factors, it follows from a theorem of Hurewicz [10] that $V_{p}$, and therefore $M_{1}$, is of dimension $n-s$. In addition, $M_{1}$ is locally contractible: Consider an arbitrary $t \in T^{s}$; then $h\left(V_{p} \times t\right)$ is a set in $S^{n}$ which is mapped by $\phi$ homeomorphically onto $V_{p}$. Consider a small spherical neighborhood $U$ of $h(p \times t)$, contained in $\phi^{-1}\left(V_{p}\right)$, and let $W=U \cap h\left(V_{p} \times t\right)$. Then $\phi(W)$ is a neighborhood of $p$, contained in $V_{p}$. The set $W$ is obviously contractible in $U$, and $\phi$ maps the contraction of $W$ into a contraction of $\phi(W)$, taking place in $\phi(U)$, and so in $V_{p}$. It follows that the singular groups of $M_{1}$ are isomorphic with the Cech groups of $M_{1}$ (cf. [11, p. 107]), and so they vanish in dimensions above that of $M_{1}$. We have found:

$$
H^{i}\left(M_{1}\right)=0 \quad \text { for } i>n-s,
$$

and as noted above, this suffices to prove Theorem 1 under the weakened hypothesis. In the case of Theorem 2, assuming $M_{2}$ separable metric only, one sees, by the same reasoning as above, that $M_{2}$ is locally compact, locally contractible, and of dimension $n-s$. In order 
to establish equation $\left(12^{\prime}\right)$ (and with it Theorem 2 under the weakened hypothesis) one would have to have a theorem to the effect that in a separable metric, locally compact, locally contractible space the singular homology groups and the compact Cech groups are isomorphic, and so vanish for dimensions above the dimension of the space.

(b) Theorem 2 verifies in a special case the conjecture that $E^{n}$ cannot be fibered with any compact fiber (cf. $[12 ; 13]$ ).

(c) Theorem 1 gives a topological proof for the fact (which is useful in determining which spheres are group manifolds (cf. [14])): If a sphere is a Lie group then its rank is 1 . The rank is the dimension of a maximal abelian subgroup; such a subgroup is homeomorphic with a torus $T^{s}$, and the cosets of the subgroup give a fiber decomposition of the group.

\section{REFERENCES}

1. N. E. Steenrod, The classification of sphere bundles, Ann. of Math. vol 45 (1944).

2. H. Hopf and M. Rueff, Über faserungstreue Abbildungen der Sphären, Comment. Math. Helv. vol. 11 (1938-1939).

3. W. Hurewicz and N. E. Steenrod, Homotopy relations in fiber spaces, Proc. Nat. Acad. Sci. U.S.A. vol. 27 (1941).

4. B. Eckmann, Zur Homotopietheorie gefaserter Räume, Comment. Math. Helv. vol. 14 (1941-1942).

5. S. Eilenberg and S. MacLane, Relations between homology and homotopy groups of spaces, Ann. of Math. vol. 46 (1945).

6. G. W. Whitehead, On products in homotopy groups, Ann. of Math. vol. 47 (1946).

7. J. L. Kelley and E. Pitcher, Exact homomorphism sequences in homology theory, Ann. of Math. vol. 48 (1947).

8. H. Hopf, Zur Algebra der Abbildungen von Mannigfaltigkeiten, J. Reine Angew. Math. vol. 103 (1930).

9. G. T. Whyburn, Analytic topology, Amer. Math. Soc. Colloquium Publications, vol. 28, New York, 1942.

10. W. Hurewicz, Sur la dimension des produits cartesiens, Ann. of Math. vol. 36 (1935).

11. S. Lefschetz, Topics in topology, Princeton, 1942.

12. D. Montgomery and $H$. Samelson, Fiberings with singularities, Duke Math. J. vol. 13 (1946).

13. G. S. Young, Compact fiberings of the plane, Bull. Amer. Math. Soc. vol. 53 (1947).

14. H. Samelson, Über die Sphären, die als Gruppenmannigfaltigkeiten auftreten, Comment. Math. Helv. vol. 13 (1940-1941).

INSTITUTE for Advanced Study,

UNIVERSITE DE LAUSANNE, UNIVERSITY OF MICHIGAN, AND

BROWN UNIVERSITY 